\title{
Review
}

\section{Ocular manifestations of malignant hemopathies : A case based pictorial review.}

\section{Les manifestations oculaires des hémopathies malignes: Une revue iconographique de cas cliniques.}

Khallouli Asma 1,2, Saidane Rahma 1,2, Choura Racem *1,2, Khelifi Khaled 1,2, Maalej Afef 1,2, Rannen Riadh1,2.

\begin{tabular}{|c|c|}
\hline \multirow{2}{*}{$\begin{array}{l}\text { 1: Department of ophthalmology } \\
\text { Military Hospital Tunis, Tunisia } \\
\text { 2: College of medicine Tunis Tunisia } \\
\text { * Corresponding author } \\
\text { Correspondence to: } \\
\text { choura.racem @gmail.com } \\
\text { Publication data: } \\
\text { Submitted: March } 23,2020 \\
\text { Accepted: May } 28,2020 \\
\text { Online: June } 30,2020\end{array}$} & Résumé \\
\hline & $\begin{array}{l}\text { Les hémopathies malignes constituent un groupe hétérogène de pathologies qui se développent } \\
\text { à partir des cellules d'origine hématopoïétique. L'atteinte oculaire est rare mais polymorphe. Elle } \\
\text { peut être due à une infiltration directe par les cellules néoplasiques ou secondaire aux anomalies } \\
\text { de la crase sanguine. L'atteinte ophtalmologique au cours des hémopathies malignes peut être } \\
\text { révélatrice de la maladie ou indiquer une rechute. Elle est associée à un pronostic péjoratif et } \\
\text { nécessite une prise en charge urgente. L'objectif de notre étude était de décrire les différentes } \\
\text { manifestations ophtalmologiques au cours des hémopathies malignes. }\end{array}$ \\
\hline \multirow{3}{*}{$\begin{array}{l}\text { This article was subject to full } \\
\text { peer-review. }\end{array}$} & $\begin{array}{l}\text { Mots clés } \\
\text { Hémopathies malignes, Hémorragies à centre blanc, Tâches de ROTH, Rétinopathie de stase, } \\
\text { Occlusion de la veine centrale de la rétine, Exophtalmie, Paralysie oculomotrice. }\end{array}$ \\
\hline & Abstract \\
\hline & $\begin{array}{l}\text { Malignant hemopathies are a group of diseases related to hematopoiesis disorders with malignant } \\
\text { behavior and evolution. Ocular involvement is rare and polymorphous. It may be due to direct } \\
\text { infiltration of the eye by neoplastic cells or secondary to hematologic abnormalities. } \\
\text { The correlation between hematologic abnormalities and ophthalmological manifestations has been } \\
\text { well demonstrated. Ophthalmological symptoms may reveal the disease or indicate a relapse. It } \\
\text { is an independent prognostic indicator. The aim of this study was to highlight the characteristics } \\
\text { of ocular disorders during malignant hemopathies. }\end{array}$ \\
\hline 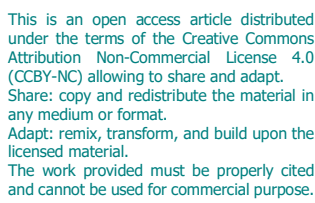 & $\begin{array}{l}\text { Key words } \\
\text { Malignant hemopathies, White-centered hemorrhages, ROTH spots, viscosity-related Retinopathy, } \\
\text { Central retinal vein occlusion, Exophthalmia, Oculomotor nerve palsy. }\end{array}$ \\
\hline
\end{tabular}

Citation: Khallouli A, Saidane R, Choura R, Khelifi K, Maalej A, Rannen R. Ocular manifestations of malignant hemopathies : A case based pictorial review.Jr.med.res. 2020; 3(2):3-10. Khallouli et al @ All rights are reserved. https://doi.org/10.32512/jmr.3.2.2020/3.10 


\section{Introduction}

Les hémopathies malignes sont des affections qui se développent à partir de cellules d'origine hématopoïétique. Elles constituent un groupe de tumeurs issues de la transformation maligne de cellules souches ou de leurs dérivés à différents stades de différenciation. La grande diversité vue dans ce groupe de maladies reflète la complexité du processus de l'hématopoìèse [1].

Les manifestations ophtalmologiques font partie des localisations secondaires des hémopathies malignes. Elles sont polymorphes, pouvant toucher toutes les structures oculaires [2]. Le but de notre étude était de décrire les différentes manifestations ophtalmologiques au cours des hémopathies malignes à travers une série de 5 observations.

\section{Diagnostic des hémopathies malignes}

Avant l'avènement de la biopsie de la moelle osseuse (BOM), l'avis des ophtalmologistes était systématiquement sollicité pour aider au diagnostic des hémopathies malignes. Malgré sa complexité, la classification des tumeurs hématopoïétiques proposée par l'Organisation mondiale de la santé (OMS) offre aux praticiens une définition précise des différentes entités en prenant compte des données anatomopathologiques et génétiques. Les hémopathies malignes sont subdivisées en deux grands groupes selon les lignées cellulaires impliquées : les hémopathies malignes myéloïdes et les hémopathies malignes lymphoïdes $[1,3]$. Les proliférations myéloïdes sont classées en quatre catégories : les syndromes myéloprolifératifs chroniques, les syndromes myélodysplasiques (SMD), les leucémies aigues myéloïdes (LAM) et les entités intermédiaires. Les hémopathies malignes du tissu lymphoïde prennent naissance à partir des cellules lymphoïdes $B$ ou $T$. Au sein des proliférations lymphoïdes, il faut distinguer les proliférations développées à partir de cellules immatures donnant des leucémies aigues (LAL B ou LAL T), des proliférations développées à partir des cellules matures qui sont de loin les plus fréquentes, à savoir la leucémie lymphoïde chronique B (LLC) et le myélome multiple (MM).

\section{Epidémiologie de l'atteinte oculaire}

La prévalence de l'atteinte oculaire au cours des leucémies peut varier entre $9 \%$ à $90 \%$ selon les études $[4,5]$. Cette grande disparité peut être due aux différences dans les groupes d'âge des populations étudiées mais aussi en raison du caractère temporaire des signes ophtalmiques. Cette atteinte est due principalement à deux mécanismes : une infiltration directe dite primaire par les cellules néoplasiques ou liée à une atteinte secondaire aux anomalies de la crase sanguine, aux traitements utilisés, à l'immunosuppression et aux infections opportunistes [6].
Dans la littérature, l'atteinte ophtalmologique secondaire ou indirecte est de loin la plus fréquente. Une prédominance liée au sexe et au mode d'évolution a été décrite. Ces anomalies étaient surtout observées chez les patients adultes de sexe masculin porteurs de LAM [7].

\section{Caractéristiques anatomocliniques}

Toutes les structures peuvent être touchées [8]. L'infiltration leucémique directe peut toucher l'orbite, le segment antérieur, I'uvée et le nerf optique. Elle peut causer une exophtalmie, une paralysie des paires crâniennes ou un œdème papillaire. Les manifestations ophtalmiques indirectes les plus communes sont les hémorragies rétiniennes, pré-rétiniennes ou dans le vitré et les anomalies veineuses rétiniennes. Chez certains patients atteints de leucémie, les signes ophtalmologiques peuvent être au premier plan aidant ainsi au diagnostic précoce de la maladie [9]. Ces signes peuvent disparaitre après le début du traitement et peuvent réapparaître lors de la rechute de la maladie.

Les manifestations oculaires du segment postérieur sont les plus rapportées dans la littérature et les hémorragies rétiniennes sont les lésions les plus fréquentes $[5,8,10]$.

Le terme « rétinopathie leucémique » est utilisé pour décrire les manifestations rétiniennes de l'anémie, thrombocytopénie et hyperviscosité plutôt que l'infiltration leucémique directe. Les hémorragies rétiniennes peuvent survenir à tous les niveaux surtout au niveau du pôle postérieur. Certaines hémorragies peuvent avoir un aspect à centre blanc contenant des cellules leucémiques, agrégats plaquettaires et fibrine ou emboles septiques appelés « tâches de Roth ». En outre, les nodules cotonneux ont été décrits au cours des leucémies, associés à des occlusions artériolaires. L'hyperleucocytose extrême peut conduire à un ralentissement au niveau de la microcirculation et par conséquence une diminution de perfusion périphérique et néovascularisation. Ceci est notamment observé chez les patients atteints de LMC $[11,12]$. Les microanévrysmes rétiniens périphériques ont été retrouvés dans environ la moitié des patients atteints de leucémie chronique à un stade terminal [13].

Tous les patients avec hémorragies rétiniennes et/ou des nodules cotonneux avaient un taux d'hémoglobine bas. Par ailleurs, les taches de ROTH étaient associées à un taux de leucocytes élevée [14]. Kezuka et al ont étudié les complications oculaires au cours des SMD. Ils ont conclu que la fréquence des atteintes oculaires chez ces patients n'était pas significativement différente de celle des patients atteints de LAM. Par ailleurs, la fréquence des hémorragies rétiniennes au cours des SMD dépendait considérablement de la sévérité de la thrombopénie (Observation1)[15]. L'infiltration choroïdienne a été surtout décrite dans les séries autopsiques. Il s'agit de décollements séreux rétiniens avec des infiltrations 


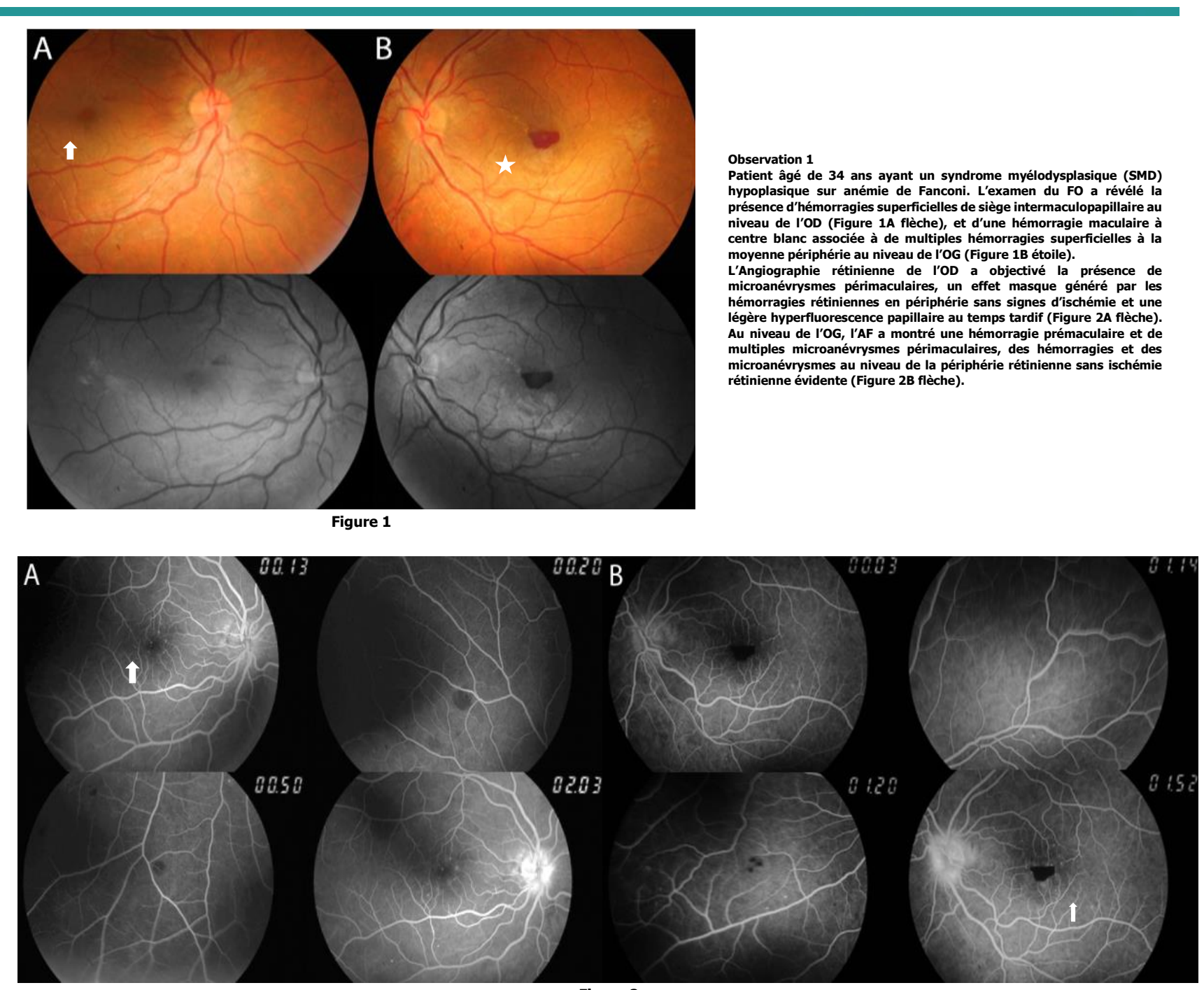

Figure 2

choroïdiennes des irrégularités de l'épithélium pigmentaire sus-jacent.

Une diminution du flux sanguin et un épaississement choroïdien ont été rapportés dans une étude récente.

Ces altérations étaient très probablement secondaires à une ischémie par compression de la choriocapillaire par les cellules néoplasiques [16]. L'infiltration leucémique du nerf optique est rare.

L'hyperviscosité sanguine par hyperleucocytose majeure peut se présenter sous forme de rétinopathie de stase bilatérale, avec dilatation et tortuosité de l'ensemble des veines rétiniennes donnant un aspect mimant une occlusion de la veine centrale sans occlusion évidente appelée aussi fundus paraproteinamucus. Elle est liée au ralentissement circulatoire qui affecte de manière prépondérante le secteur veineux et à un certain degré d'hyperviscosité, une occlusion veineuse véritable peut survenir. L'invasion du nerf optique rétrobulbaire semble beaucoup plus dévastatrice pour la vision que l'invasion leucémique de la tête du nerf optique, bien que les deux peuvent coexister (Observation 2) [17-19].

\section{Implications pronostiques}

Il a été démontré que l'atteinte ophtalmique est associée à un mauvais pronostic dans les leucémies aiguës de l'enfant. Par conséquent, il est important d'effectuer un examen ophtalmologique systématique au moment du diagnostic chez les patients atteints de leucémie [20]. L'atteinte orbitaire est considérée comme atteinte du système nerveux central (SNC).

Elle nécessite une prise en charge urgente et spécialisée. La LAL est associée à un risque d'atteinte oculo-méningée plus important que celui de la LAM, ce qui explique le traitement prophylactique systématique instaure aux patients et qui repose sur les injections intrathécales répétées de méthotrexate avec renforcement de la 

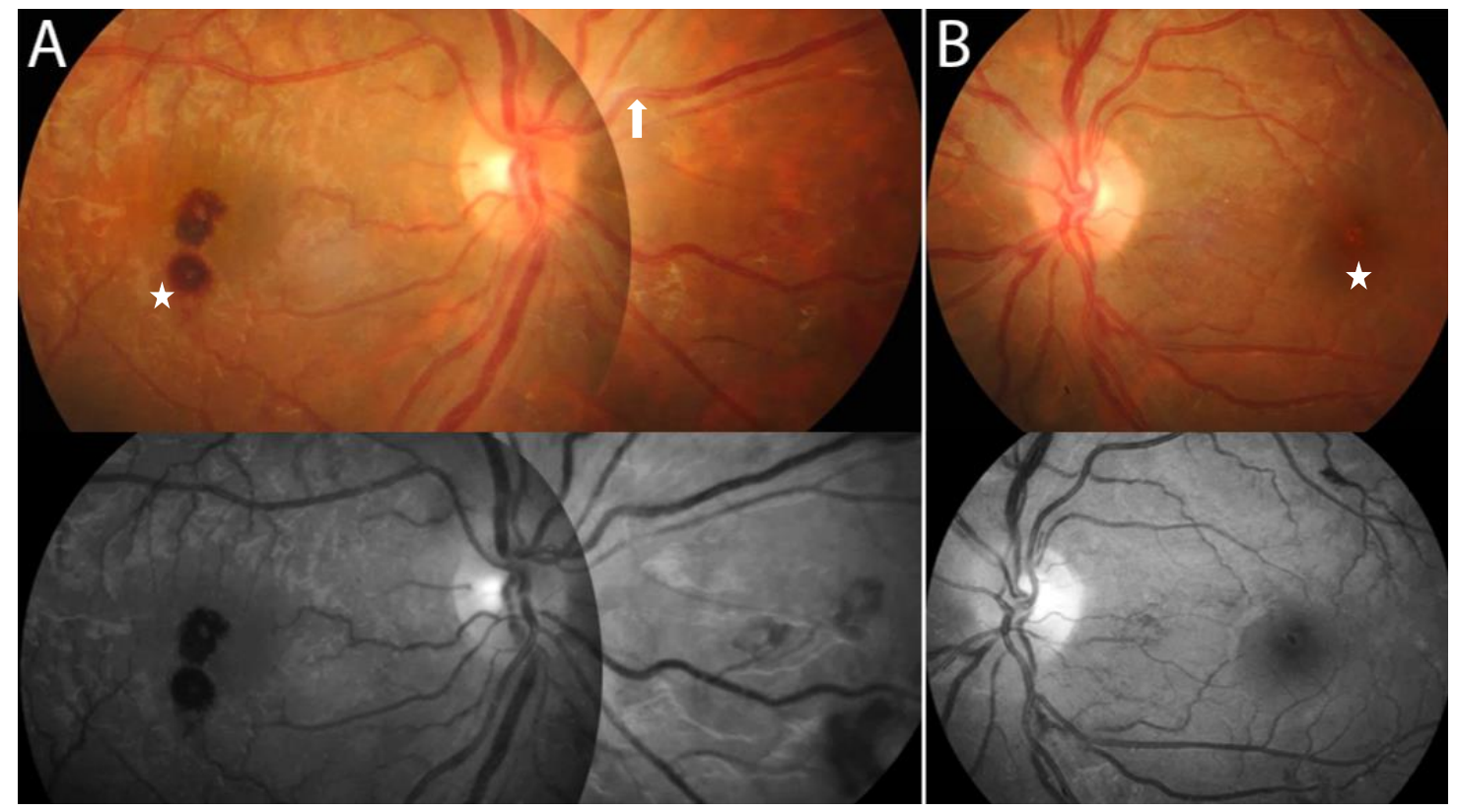

Figure 3

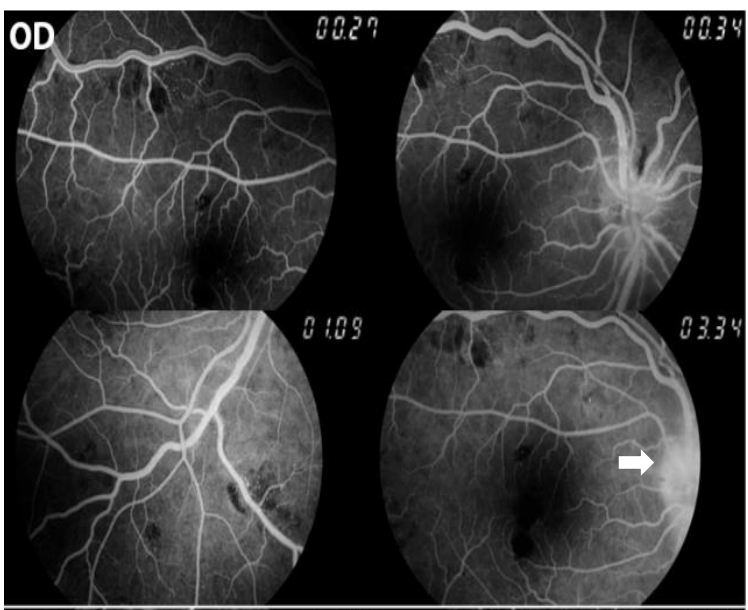

Observation 2

Patient âgé de 26 ans, présentant une leucémie myéloïde chronique (LMC) avec un transcrit BCR-ABL de type b2a2 sous traitement par les inhibiteurs de la tyrosine kinase. Son histoire montre de multiples accidents thromboemboliques systemiques et une thrombopenie profonde. L'examen du segment antérieur a noté la présence d'une dilatés et cous conjonctivale au nive dilatées et tortueuses au niveau des deux yeux, deux hémorragies maculaires rondes à (Figure 3A) as quectaues hémorragies superficielles se siège interm temporal au niveau de l'OG (Figure 3B) L'évolution à deux mois a été marquée par une transformation de la LMC en leucémie aigüe myélö̈ (LAM) avec une hyperleucocytose majeure $(180.000 / \mathrm{mm} 3)$ et une thrombopénie à $75.000 / \mathrm{mm}^{3}$. L'examen du FO de contrôle a montré une aggravation des hémorragies rétiniennes et des dilatations veineuses avec apparition d'un œè̀me papillaire bilatéral (Figure 4). L'AF a montré au niveau des deux yeux, des veines dilatées et tortueuses, un effet masque généré par les hémorragies rétiniennes au niveau du pôle postérieur et de la périphérie rétinienne, des zones hypo-fluorescentes jouxtant les hémorragies rétiniennes correspondant à des zones de non-perfusion, une hyperfluorescence papillaire tardive en rapport avec l'œdème papillaire (Figure5). Il s'agissait d'une rétinopathie de stase liée à I'hyperviscosité sanguine (hyperleucocytose majeure) ou « fundus paraproteinaemicus» au niveau des deux yeux. Le patient a été mis sous chimiothérapie intensive avec une réponse clinique médiocre.

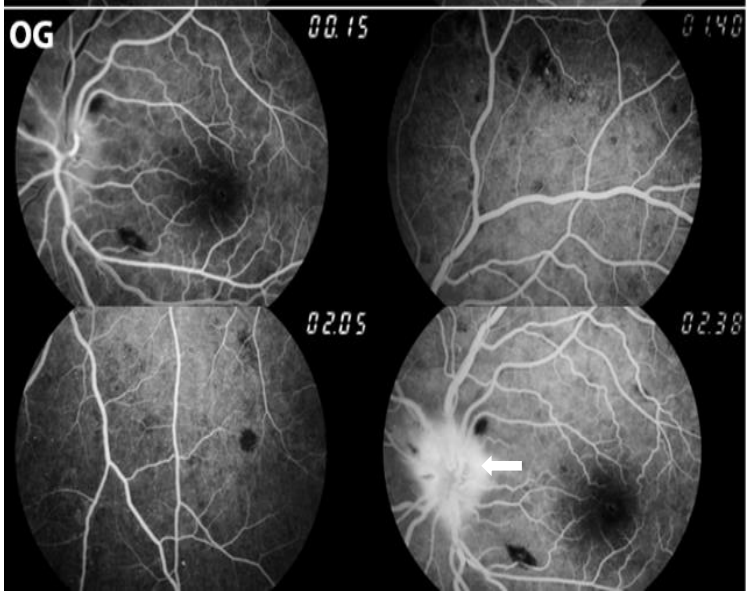

Figure 5

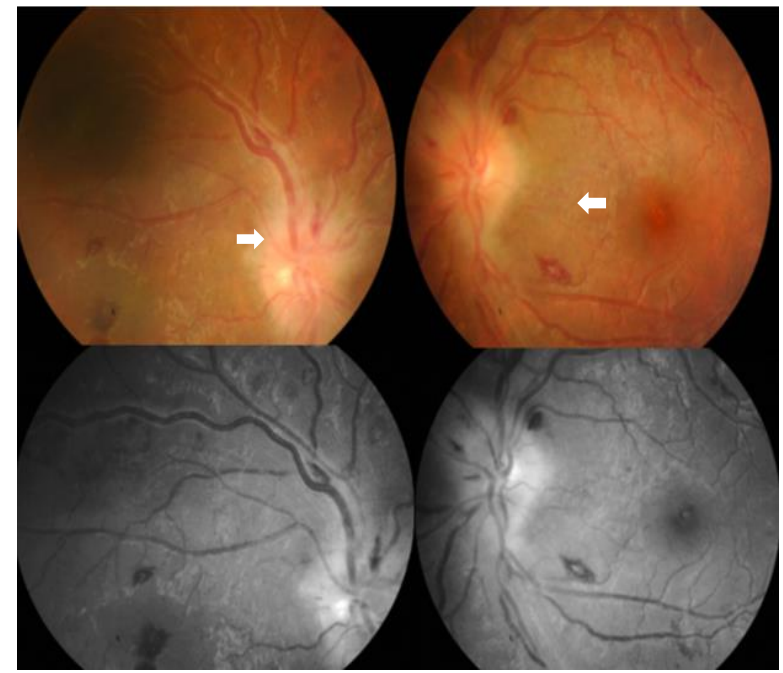

Figure 4

Citation: Khallouli A, Saidane R, Choura R, Khelifi K, Maalej A, Rannen R. Ocular manifestations of malignant hemopathies : A case based pictorial review.Jr.med.res. 2020; 3(2):3-10. Khallouli et al (c) All rights are reserved. 
chimiothérapie systémique (Observation 3)[21]. Plusieurs études ont été menées pour évaluer la relation entre la présence de manifestations oculaires au cours de la leucémie et le taux de survie. Il a été rapporté que $80 \%$ des enfants décédaient dans les 10 mois suivant le diagnostic de l'atteinte oculaire dans la leucémie aiguë [22].

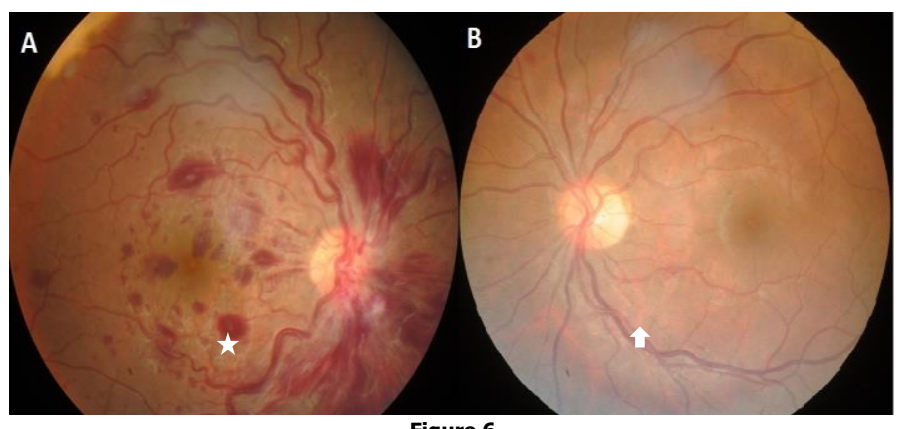

Figure 6

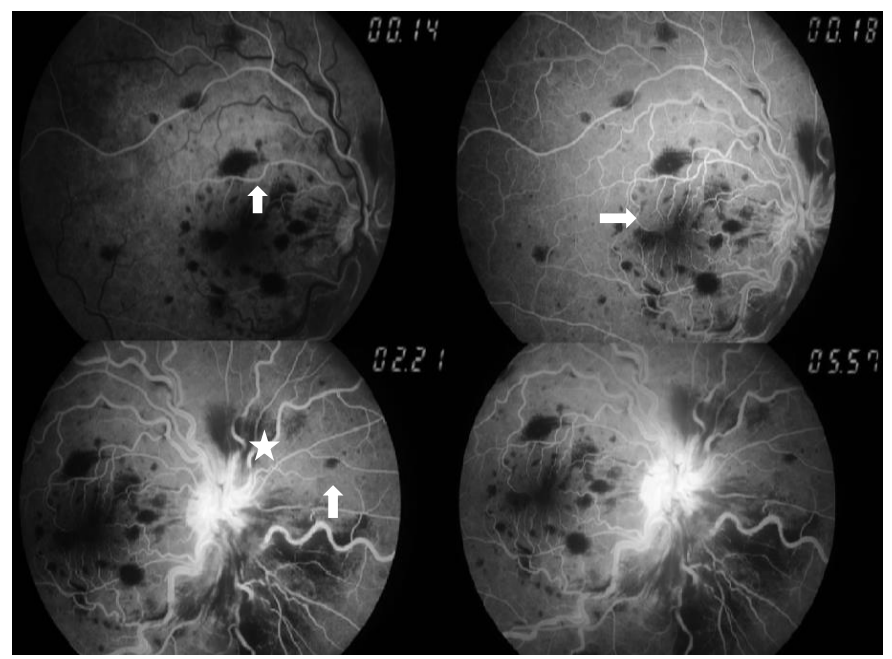

Figure 7

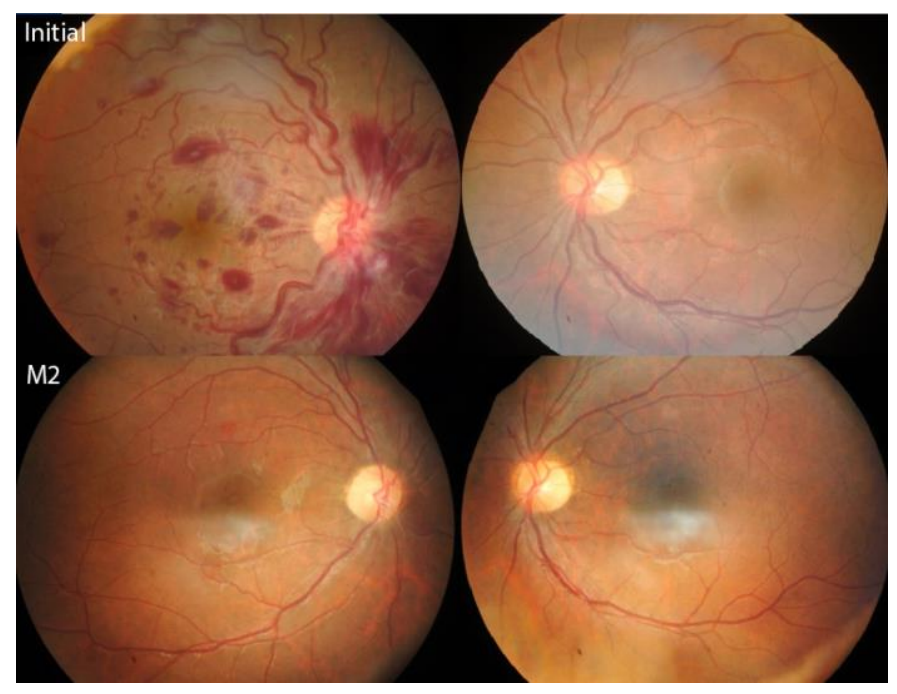

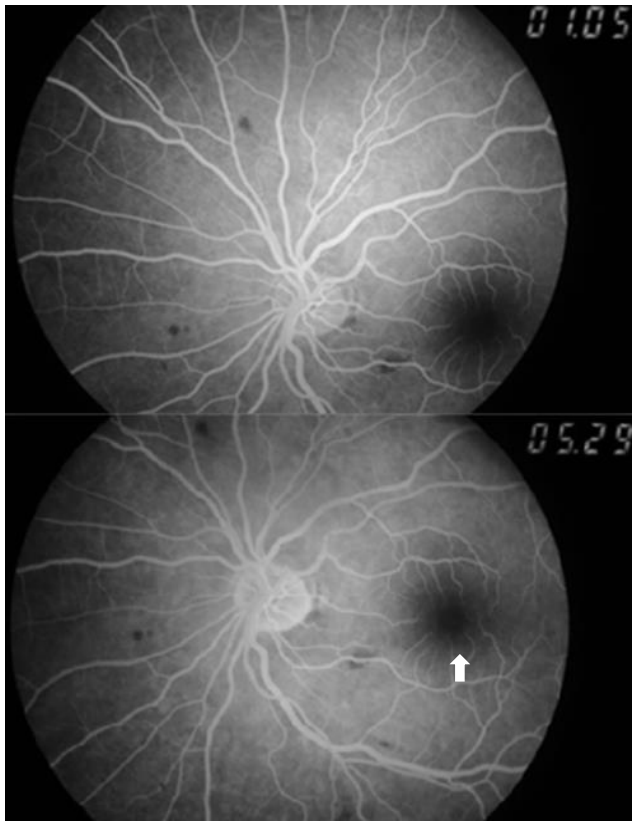

Fiaure 8

Observation 3

Patient de 19 ans ayant une leucémie aigüe lymphoblastique $B$ ayant un flou visuel au niveau de son OD. L'examen a trouvé une acuité visuelle conservée à 10/10ème au niveau des deux yeux, un segment antérieur et un tonus oculaire normaux. L'examen du FO a montré au niveau de I'OD des veines dilatées et tortueuses, de multiples hémorragies péri-papillaires superficielles en flammèches et un œdème papillo-rétinien ainsi qu'une hémorragie périmaculaires à centre blanc (Figure 6a). Au niveau de I'œil gauche (OG), nous avons objectivé la présence de quelques hémorragies superficielles de petite taille en nasal (Figure $6 b$ flèche).

petite taille en nasal (Figure $6 \mathrm{~b}$ flèche).
L'angiographie rétinienne à la fluorescéine (AF) a mis en évidence au niveau L'angiographie rétinienne à la fluorescéine (AF) a mis en évidence au niveau
de I'OD un allongement du temps artérioveineux, un retard de remplissage veineux, un effet masque en rapport avec les hémorragies rétiniennes et une hyperfluorescence papillaire tardive correspondant à l'œdème (Figure 7). Au niveau de I'OG, I'AF a montré un effet masque des hémorragies rétiniennes en nasal sans signes d'ischémie (Figure 8). La tomographie en cohérence optique Spectral Domain (OCT-SD) de la macula était sans anomalies aux niveaux des deux yeux.

Il s'agissait d'une occlusion de la veine centrale de la rétine de l'OD dans sa forme œdémateuse. Une polychimiothérapie a permis d'obtenir une rémission clinique en deux mois avec normalisation des examens optiques (Figure 9).

Le taux de survie à 5 ans des patients présentant des manifestations ophtalmiques était nettement inférieur à celui de ceux sans manifestations ophtalmologiques selon certains auteurs [23].

\section{Particularités de l'atteinte myélomateuse} L'origine myélomatose représente moins de $1 \%$ de toutes les tumeurs orbitaires [24]. Toutes les structures oculaires peuvent être touchées. Des infiltrations orbitaires, conjonctivales, uvéales, du sac et des glandes lacrymales ont été décrites [25]. Ces structures peuvent être atteints soit par infiltration directe par les cellules plasmocytaires ou les dépôts d'Immunoglobulines, soit secondaire aux anomalies hématologiques (observations 4 et 5 ).

Des atteintes du segment postérieur ont été également observées à type de choriorétinopathie (infiltrat de la choroïde, des dilatations et des 
tortuosités veineuses, des occlusions veineuses, des hémorragies rétiniennes, des micro-anévrysmes, des nodules cotonneux) et d'anomalies neuro-ophtalmiques (parésie musculaire extra-oculaire ou infiltrat direct myélomateux, œdème papillaire).

L'atteinte orbitaire myélomatose sans destruction osseuse locale ou atteinte oculaire associée est extrêmement rare et l'invasion musculaire est encore plus rare [26]. Elle est révélatrice de la maladie dans seulement $25 \%$ des cas [27]. Dans différentes revues de la littérature, le délai entre le diagnostic de myélome multiple et l'atteinte orbitaire était en moyenne de 17,6 mois. Cette atteinte était le premier signe de récurrence de la maladie systémique dans $9,6 \%$ cas.

L'exophtalmie était le motif principal de consultation (81\%) suivie par la baisse de l'acuité visuelle (23\%), la diplopie (23\%), l'œdème palpébral (21\%) et le ptosis $(13 \%)$. Ces manifestations étaient souvent unilatérales (88\%) [28].

L'imagerie orbitaire en particulier I'IRM présente un intérêt majeur dans le bilan lésionnel afin de mieux visualiser les structures atteintes. Mais c'est l'examen anatomopathologique des biopsies qui confirme l'infiltration plasmocytaire. La présence de localisations extra-médullaires au cours du MM indique un mauvais pronostic.

Les métastases orbitaires sont notamment associées à des taux de survie médiocres par rapport aux autres localisations secondaires [29].

La survie globale chez les patients présentant une récidive myélomateuse sous forme d'atteinte orbitaire est de 12 mois en l'absence de rechute hématologique. Ce taux est moindre en cas de rechute hématologique associée [24]. Par ailleurs il a été montré que les MM à IgG sont associés à un risque accru d'atteinte orbitaire [30].

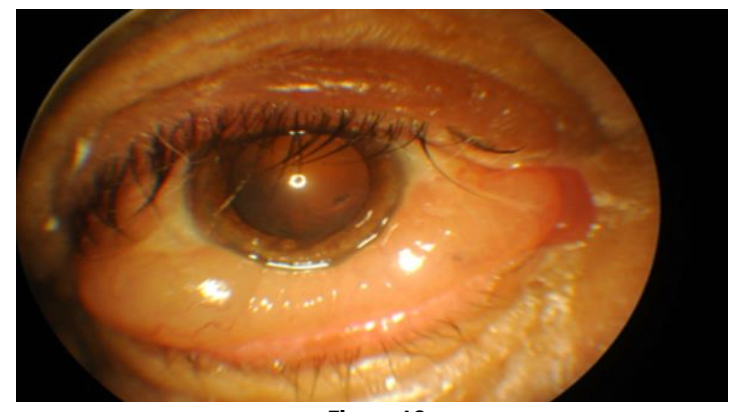

Figure 10

Observation 4

Patient âgé de 74 ans suivi pour myélome multiple (MM) à IgA à chaînes légères kappa ne répondant que partiellement au traitement. L'évolution a été marquée par I'apparition récente d'une exophtalmie de l'OD. A l'examen, le patient avait une meilleure acuité visuelle corrigée (MAVC) à 8/10ème, une limitation de l'abduction, un œdème palpébral, une exophtalmie axile non pulsatile, un chémosis (Figure 10) un réflexe photomoteur direct et consensuel présent, avec un examen du FO sans anomalies. L'examen de I'OG était sans particularités. Une imagerie orbitaire (Tomodensitométrie et Imagerie par résonance magnétique) a montré la présence d'un épaississement des muscles droit latéral, supérieur et médial à droite responsable d'une exophtalmie droite grade I avec une prise de contraste hétérogène du corps de l'os sphénoïde évoquant en premier lieu une localisation myélomateuse (Figure 11). Le dosage des IgG 4 était normal. Une biopsie musculaire a mis en évidence l'infiltration plasmocytaire des muscles orbitaires et la rechute du myélome fut confirmée. Une chimiothérapie plus agressive était instaurée avec une régression progressive de l'exophtalmie.

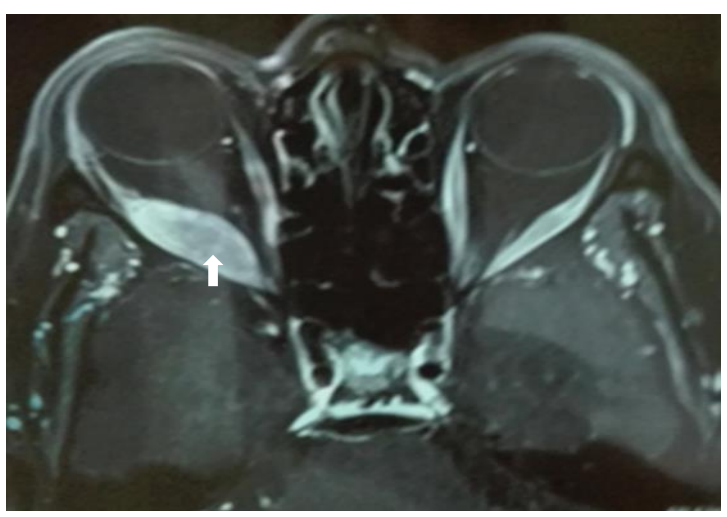

Figure 11

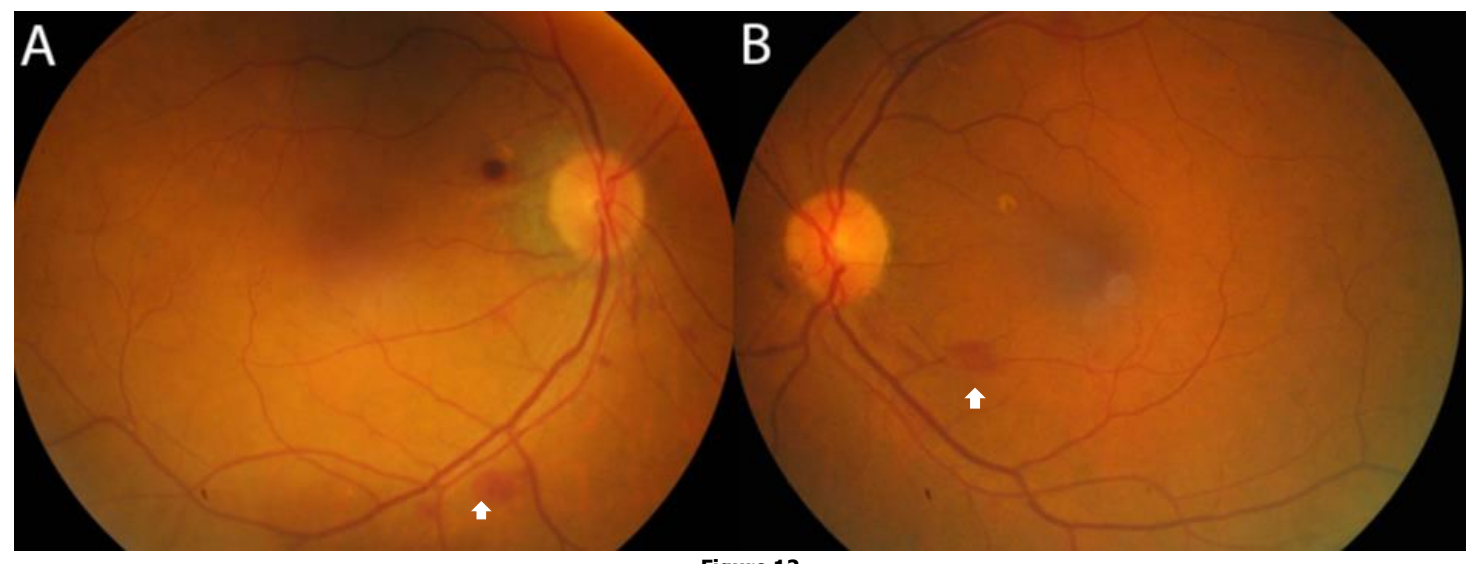

Figure 12 


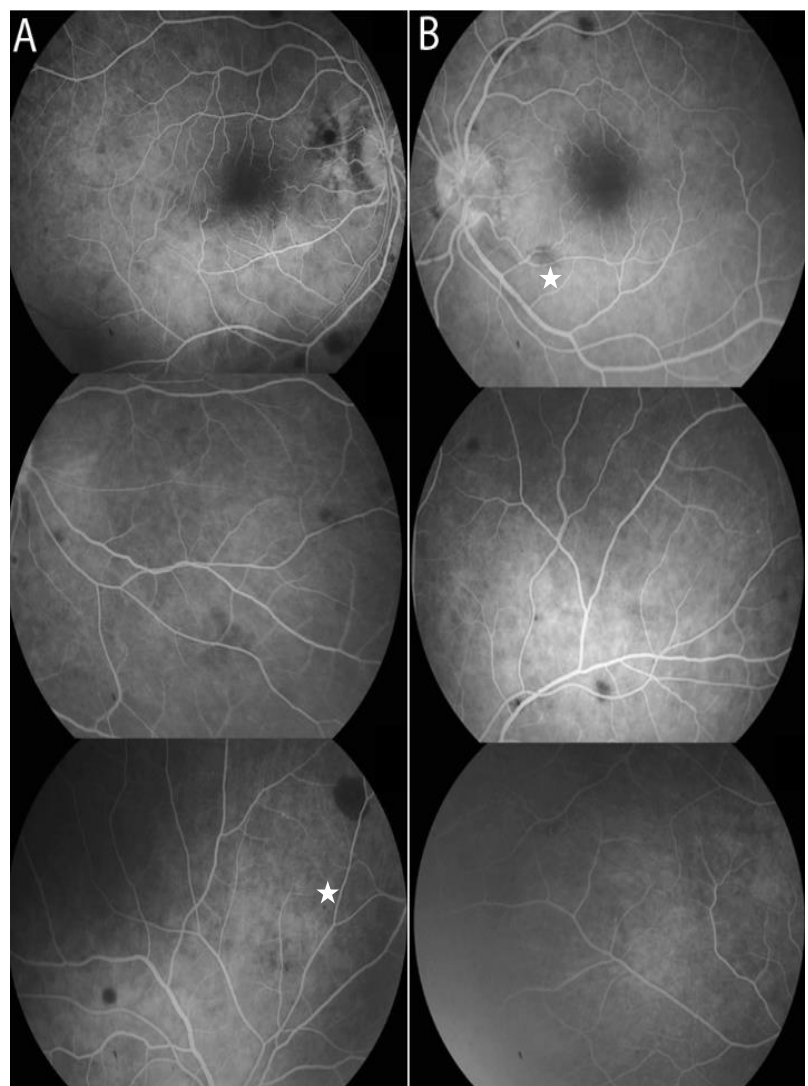

Figure13

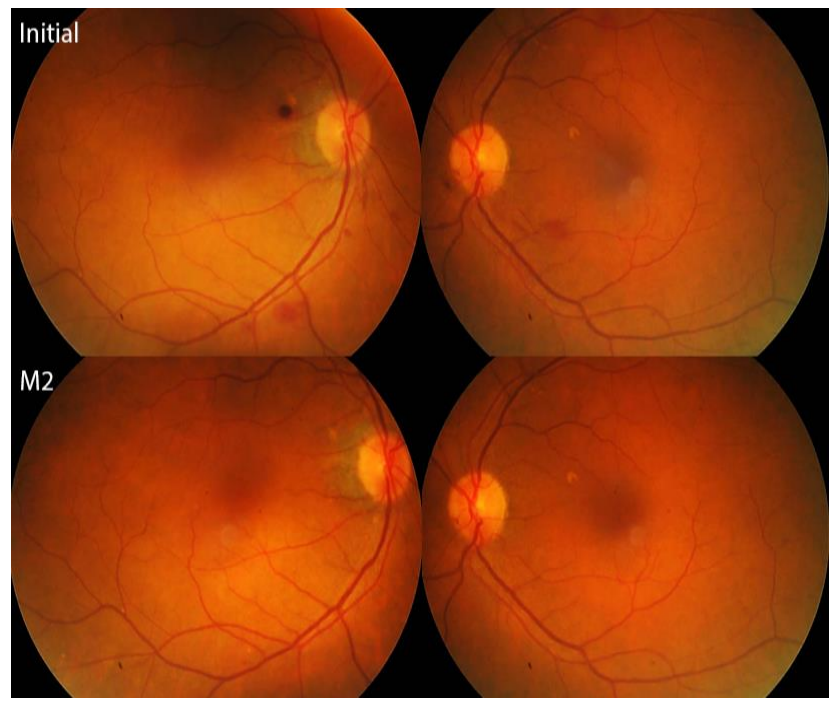

Figure 14

Observation 5

Patient âgé de 62 ans, suivi pour myélome multiple à IgG kappa avec une leuco-thrombopénie de découverte récente et flou visuel bilatéral progressif. L'examen ophtalmologique a montré une MAVC à 7/10ème au niveau des deux yeux avec un segment antérieur normal. Après dilatation pupillaire, nous avons noté la présence d'une cataracte cortico-nucléaire et souscapsulaire peu dense bilatérale. L'examen du FO au niveau de I'OD a objectivé une zone d'altération de l'épithélium pigmentaire de siège péri-papillaire, une hémorragie profonde ronde juxta-papillaire et des hémorragies superficielles multifocales, en cocarde, siégeant surtout en temporal inférieur (Figure 12A étoile). L'examen du FO au niveau de l'OG a retrouvé de multiples hémorragies superficielles en flammèche (Figure 12B).

L'AF de I'OD a mis en évidence un effet fenêtre de siège péri-papillaire correspondant aux L'AF de l'OD a mis en évidence un effet fenêtre de siège péri-papillaire correspondant aux
zones d'atrophie de l'épithélium pigmentaire, et un effet masque correspondant à de multiples hémorragies rétiniennes superficielles et profondes. Les clichés tardifs n'ont pas montré de territoires d'ischémie rétinienne (Figure 13A). Au niveau de l'OG, nous avons noté la présence d'hémorragies rétiniennes superficielles diffuses, sans dilatation ni tortuosité veineuse et en l'absence de signes d'hypoperfusion rétinienne (Figure 13B).

Le renforcement du protocole thérapeutique a permis d'obtenir une bonne réponse clinique. Et une disparition des hémorragies rétiniennes au bout de deux mois (Figure 14).

\section{Points essentiels}

- L'atteinte ophtalmologique au cours des hémopathies malignes est polymorphe et peut être révélatrice de la maladie ou annoncer une rechute. L'examen ophtalmologique peut constituer un moyen de surveillance de I'hémopathie fiable et reproductible.

- L'atteinte oculaire est souvent asymptomatique. Les signes oculaires au cours d'une hémopathie maligne sont variables et non spécifiques. Un examen ophtalmologique systématique est nécessaire au début de la maladie, à répéter au moindre signe d'appel.

- L'atteinte ophtalmologique est associée à un mauvais pronostic visuel et à une survie liée à l'hémopathie significativement raccourcie.

- L'atteinte oculaire est sous diagnostiquée. Il faut savoir évoquer une hémopathie maligne devant une hémorragie conjonctivale atypique et inexpliquée.

- Le traitement des lésions oculaires est une concertation multidisciplinaire. Le traitement des lésions oculaires rejoint celui de l'hémopathie. Ces lésions restent réversibles une fois diagnostiquées et traitées précocement. Cependant les récidives sont fréquentes vu le comportement agressif de ce type de tumeurs.

- La localisation oculaire myélomateuse est très polymorphe et toujours de mauvais pronostic.

\section{Conflits d'intérêt : Aucun}




\section{Références}

[1] Sabattini E, Bacci F, Sagramoso C, Pileri SA. WHO classification of tumours of haematopoietic and lymphoid tissues in 2008: an overview. Pathologica. 2010;102(3):83-87.

[2]Jarrett WF, Mackey $L$. Neoplastic diseases of the haematopoietic and lymphoid tissues. Bull World Health Organ. 1974; 50:21-34.

[3] Arber DA, Orazi A, Hasserjian R, Thiele J, Borowitz MJ, Michelle M Le Beau MM, et al. The 2016 revision to the World Health Organization classification of myeloid neoplasms and acute leukemia. Blood. $2016 ; 127: 2391-2405$.

[4]Silva JR Jr, Hayashi D, Yonenaga T, Fukuda K, Genant HK, Lin C, et al. MRI of bone marrow abnormalities in hematological malignancies. Diagn Interv Radiol. 2013;19:393-99.

[5]Orhan B, Malbora B, Akça Bayar S, Avcı Z, Alioğlu B, Özbek N. Ophthalmologic Findings in Children with Leukemia: A Single-Center Study. Turk J Ophthalmol. 2016;46:62-7.

[6] Gawai D, Jhavar S, Patil S: Orbital and ocular manifestations of acute and chronic leukemia. Int J Health Sci Res. 2016;6:61-4.

[7]Koshy J, John MJ, Thomas S, Kaur G, Batra N, Xavier W. Ophthalmic manifestations of acute and chronic leukemias presenting to a tertiary care center in India. Indian J Ophthalmol. $2015 ; 63: 659-64$.

[8] Chaabani L, Doulami K. White-centred retinal hemorrhage revealing acute leukemia. Tunis Med. $2019 ; 97: 822-25$.

[9] Soman S, Kasturi N, Srinivasan R: Ocular manifestations in leukemias and their correlation with hematologic parameters at a tertiary care setting in south India. Ophthalmol Retina. 2018;2:17-23.

[10]Almalki AMJ, Alotaibi FA, Jabr HF, Mastan AR. Unilateral proptosis as an initial sign of acute Myeloid Leukemia in a child: A case report. Int Med Case Rep J. 2019; 12:319-23

[11]Asadi-Amoli F, Nozarian Z, Bonaki HN, Mehrtash V, Entezari S. Clinicopathologic assessment of ocular adnexal lymphoproliferative lesions at a Tertiary Eye Hospital in Iran. Asian Pac J Cancer Prev. 2016; 17:3727-31.

[12]Liu TYA, Smith BD, Mackey K, Linz MO, Scott AW. Retinal vascular changes on optical coherence tomography angiography and ultra-widefield Fluorescein Angiography in patients with chronic Leukemia. J Vitreoretin Dis. 2019; 3:420-7.

[13] Bylsma GW, Hall AJ, Szer J, West R. Atypical retinal microvasculopathy after bone marrow transplantation. Clin Exp Ophthalmol. 2001; 29:225-29.

[14]Němčanská S, Stepanov A, Němčanský J. Ophthalmic manifestations of acute leukaemias. Cesk Slov Oftalmol. 2018; 74:98-101.

[15] Kezuka T, Usui N, Suzuki E, Wakasugi K, Usui M. Ocular complications in myelodysplastic syndromes as preleukemic disorders. Jpn J Ophthalmol. 2005;49:377-83.

[16]Takita A, Hashimoto Y, Saito W, Kase S, Ishida S. Changes in blood flow velocity and thickness of the choroid in a patient with leukemic retinopathy. Am J Ophthalmol Case Rep. 2018; 12:68-72.

[17]Bouladi M, Zerei N, Bouraoui R, El Matri L. Unilateral infiltration of the optic nerve revealing relapse of an acute lymphoblastic leukemia. Tunis Med. 2019; 97:504-7.

[18]Blau-Most M, Gepstein R, Rubowitz A. Bilateral simultaneous central retinal vein occlusion in hyperviscosity retinopathy treated with systemic immunosuppressive therapy only. Am J Ophthalmol Case Rep. 2018; 12:49-51.

[19]Kolar P. Risk factors for central and branch retinal vein occlusion: a metaanalysis of published clinical data. J Ophthalmol. 2014; 2014:724780.

[20]Reddy SC, Jackson N, Menon BS. Ocular Involvement in Leukemia. A Study of 288 Cases. Ophthalmologica. 2003; 217:441-5.

[21]Buchan J, McKibbin M, Burton T. The prevalence of ocular disease in chronic lymphocytic leukaemia. Eye (Lond). 2003; 17:27-30.

[22]Sagiv O, Thakar SD, Manning JT, et al. Prevalence of a histologic change of ocular adnexal Lymphoma in patients with a history of Lymphoma. Ophthalmic Plast Reconstr Surg. 2019; 35:243-46.

[23]Ohkoshi K, Tsiaras WG. Prognostic importance of ophthalmic manifestations in childhood leukaemia. $\mathrm{Br}$ J Ophthalmol. 1992; 76:651-5.

[24]Kottler UB, Cursiefen C, Holbach LM. Orbital involvement in multiple myeloma: first sign of insufficient chemotherapy. Ophthalmologica. 2003; 217:76-8.

[25] Chin KJ, Kempin S, Milman T, Finger PT. Ocular manifestations of multiple myeloma: three cases and a review of the literature. Optometry. 2011;82:224-30.
[26] Saffra N, Gorgani F, Panasci D, Kirsch D. Diplopia and proptosis due to isolated lateral rectus plasmacytoma in a patient with multiple myeloma. BMJ Case Rep. 2019;12:e229178.

[27] Silva JR Jr, Hayashi D, Yonenaga T, Fukuda K, Genant HK, Lin C, et al. MRI of bone marrow abnormalities in hematological malignancies. Diagn Interv Radiol. 2013;19:393-99.

[28]Tai E, Sim SK, Haron J, Wan Hitam WH. Orbital multiple myeloma: a diagnostic challenge. BMJ Case Rep. 2017 ;2017: bcr2017220895.

[29]Orhan B, Malbora B, Akça Bayar S, Avcı Z, Alioğlu B, Özbek N. Ophthalmologic Findings in Children with Leukemia: A Single-Center Study. Turk J Ophthalmol. 2016;46:62-7.

[30]Thoumazet F, Donnio A, Ayeboua L, Brebion A. Orbital and muscle involvement in multiple myeloma. Can J Ophthalmol. 2006;41:733-6. 